\title{
The level of CD147 expression correlates with cyclophilin-induced signalling and chemotaxis
}

\author{
Alexander Trachtenberg ${ }^{1 \dagger}$, Tatiana Pushkarsky ${ }^{1 \dagger}$, Shannon Heine ${ }^{1 \dagger}$, Stephanie Constant ${ }^{1,2}$, Beda Brichacek ${ }^{1,3}$ and \\ Michael Bukrinsky ${ }^{1 *}$
}

\begin{abstract}
Background: Previous studies identified CD147 as the chemotactic receptor on inflammatory leukocytes for extracellular cyclophilins (eCyp). However, CD147 is not known to associate with signal transducing molecules, so other transmembrane proteins, such as proteoglycans, integrins, and CD98, were suggested as receptor or COreceptor for eCyp. CD147 is ubiquitously expressed on many cell types, but relationship between the level of CD147 expression and cellular responses to eCyp has never been analyzed. Given the role of eCyp in pathogenesis of many diseases, it is important to know whether cellular responses to eCyp are regulated at the level of CD147 expression.

Results: Here, we manipulated CD147 expression levels on HeLa cells using RNAi and investigated the signalling and chemotactic responses to eCypA. Both Erk activation and chemotaxis correlated with the level of CD147 expression, with cells exhibiting low level expression being practically unresponsive to eCypA.

Conclusions: Our results provide the first demonstration of a chemotactic response of HeLa cells to eCypA, establish a correlation between the level of CD147 expression and the magnitude of cellular responses to eCypA, and indicate that CD147 may be a limiting factor in the receptor complex determining cyclophilin-induced Erk activation and cell migration.
\end{abstract}

\section{Background}

Leukocyte trafficking and recruitment is a critical component of inflammation-mediated pathology. The main regulators of leukocyte trafficking are chemokines, a family of chemoattracting cytokines that control cell migration and adhesion [1]. However, other factors, in particular extracellular cyclophilins (eCyp), also induce potent chemotactic responses of immune cells (reviewed in [2]). We [3-6] and others $[7,8]$ have observed that secreted cyclophilin A (CypA) is a potent leukocyte chemoattractant in vitro and in vivo.

In studies aimed at establishing the mechanism whereby cyclophilins mediate their chemotactic activity, our group identified CD147 (also known as EMMPRIN and basigin), as the signalling receptor for eCyp [3,9]. Indeed, all human $[3,6]$ and mouse $[4,5]$ leukocytes examined to date require expression of CD147 for cyclophilin-dependent chemotaxis to occur. However, CD147 is not known to mediate

\footnotetext{
* Correspondence: mtmmib@gwumc.edu

† Contributed equally

${ }^{1}$ The George Washington University, Washington, DC 20037, USA

Full list of author information is available at the end of the article
}

signal transduction events, suggesting that other molecule (s) associated with CD147 may function as a signal-transducing co-receptor. Consistent with this notion, truncation of the cytoplasmic tail of CD147 does not abolish signalling response to eCypA [10]. The possibility of additional co-receptor is also supported by inability of eosinophils to respond to eCypA despite the fact that these cells express relatively high levels of CD147 [5]. Such signaltransducing activity may be exerted by known CD147interacting proteins, such as $\beta 1$ integrins [11,12], CD98 [13], and syndecan-1 [14], which may function as a multimolecular receptor complex [13]. Whether and how the level of CD147 expression influences activity of this complex is unknown. We recently reported that activated CD4 ${ }^{+} \mathrm{T}$ cells showed enhanced cyclophilin-mediated chemotaxis relative to resting $\mathrm{T}$ cells, that correlated with an upregulated expression of CD147 [5,6], but cell activation likely increases expression of many other proteins, including those that may be involved in eCyp-induced signalling, thus precluding reliable interpretation of this result.

The chemotactic activity of CD147 has been investigated until now only for inflammatory leukocytes.

\section{C) Biomed Central}


However, CD147 is an ubiquitously expressed protein present on almost all studied cell types, including cancer cells and stem cells $[15,16]$, suggesting that eCypinduced chemotaxis may underlie such processes as metastasis or cell development. An important question in this regard is whether such response depends on the level of CD147 expression. In this study we investigated cyclophilin-induced chemotactic activity of HeLa cells manipulated by RNAi technology to express varying levels of CD147. Our results demonstrate a direct correlation between CD147 expression and signalling and chemotactic responses to eCypA, suggesting that CD147 may be a rate-limiting component in the eCypAresponding receptor complex.

\section{Methods}

RNA interference and CD147 knockdown

siRNA (small interfering RNA) was derived from the nucleotide sequence of CD147 [17] corresponding to nucleotides 470-490. Forward 5'-GATCCCCcggctccgagagcaggttcttcaagagagaacctgctctcggagccgTTTTTGGAAA-3' and reverse 5'-AGCTTTTCCAAAAAcggctccgagagcagg ttctctcttgaagaacctgctctcggagccgGGG-3' complementary oligonucletides containing the above sequence were designed according to protocol recommended for pSUPER.retro.puro expression system (OligoEngine, Seattle, WA) by the manufacturer. A mixture of both oligonucleotides $(3 \mu \mathrm{g}$ of each) was incubated in $50 \mu \mathrm{l}$ of $100 \mathrm{mM}$ $\mathrm{NaCl}$ and $50 \mathrm{mM}$ HEPES $\mathrm{pH} 7.4$ at $90^{\circ} \mathrm{C}$ for $4 \mathrm{~min}$, and then at $70^{\circ} \mathrm{C}$ for 10 minutes. The mixture was then cooled down to $10^{\circ} \mathrm{C}$ over a period of 50 minutes. pSUPER.retro. puro vector was linearized by subsequent cleavage with HindIII and BglII. Annealed nucleotides were ligated into the linearized pSUPER.retro.puro using T4 ligase as recommended by the OligoEngine protocol creating pSuper3,4 construct. E. coli Stbl 2 competent cells (Invitrogen) were transformed with pSuper3,4 and ampicillin resistant colonies were isolated and amplified.

Purified pSuper3,4 plasmid as well as pSuper empty vector were used for transfection of exponentially growing HeLa-CD4 [18] cells. Puromycin resistant cells were examined for CD147 expression by flow cytometry. pSuper3,4transformed cell population was further enriched for cells with downregulated CD147 surface expression by removal of cells with higher CD147 expression using magnetic beads with conjugated anti-CD147 antibodies (Ancell, Bayport, MN). Remaining cell population was expanded, sorted on a flow cytometer, and several cell clones with low CD147 expression were established.

\section{Reagents}

FITC-conjugated anti-CD147 mouse monoclonal antibody was purchased from Ancell. FITC-conjugated mouse IgG1 was obtained from PharMingen (BD
Biosciences, San Jose, CA). Human recombinant cyclophilin A was purchased from Calbiochem (EMD, Rockland, MA). Human SDF-1 $\alpha$ was purchased from Peprotech (Rocky Hill, NJ). The primary anti-phosphoErk1/2 rabbit monoclonal antibody (recombinant clone AW39R) was purchased from Millipore (Billerica, MA), rabbit polyclonal antibody to Erk1/2 was from Cell Signalling Technology (Boston, MA), and the secondary antibody, anti-rabbit IgG (Horseradish Peroxidase linked $\mathrm{F}(\mathrm{ab})_{2}$ fragment), was from GE Healthcare, UK.

\section{In vitro chemotaxis assay}

Chemotactic activity was assessed using 48-well modified Boyden chambers (Neuro Probe Inc., Gaithersburg, MD) with the two compartments separated by an $8 \mu \mathrm{m}$ polyvinylpyrrolidone free polycarbonate membrane (Neuro Probe Inc.). HeLa-CD4 cells [18], at $10^{4}$ cells/well in RPMI-1640 culture medium supplemented with $1 \%$ BSA, Cohn Fraction V (Sigma-Aldrich, St. Louis, MO), were added to the upper compartment ( $40 \mu$ l volume) and medium containing recombinant human CypA (100 ng/ml) or SDF- $1 \alpha(50 \mathrm{ng} / \mathrm{ml})$ or medium alone $(25 \mu \mathrm{l})$ was added to the lower compartment. The chambers were incubated for 50 minutes at $37^{\circ} \mathrm{C}$. Then the membrane was removed, nonmigrated cells were scrapped off the upper side with PBS, and the membrane, after drying, was stained with Wright-Giemsa (Camco, Fort Lauderdale, FL) to reveal bound cells. A chemotaxis index was calculated for each test well by dividing the number of cells counted for that well by the number of cells counted in media wells.

\section{Flow cytometry}

Cells were stained with $10 \mu \mathrm{g} / \mathrm{ml}$ of FITC-conjugated anti-human CD147 mAb (Ancell, Bayport, MN) or FITCIgG1 isotype control $\mathrm{mAb}$ for $15 \mathrm{~min}$ at $4^{\circ} \mathrm{C}$, washed with cold PBS and used for analysis. Flow cytometric analysis was done using a FACSCalibur instrument and CellQuest Pro software (Becton Dickinson, San Jose, CA). To analyze the staining of CD147, cells were first gated by forward and side scatter. Live cell populations were gated upon on an acquisition plot and 20,000 events were collected from this region. An acquisition histogram plot was also assessed for this region using parameter 3 , the FL-1 detector, set to Log mode in order to detect fluorescence of the green fluorophore, FITC. The voltage of the parameter was adjusted to the relative fluorescence intensity of the high CD147 expressing cell line. All subsequent samples were acquired using the same settings. Isotype-stained cells were used as control; background staining was less than $1 \%$.

\section{Signalling analysis}

Serum-starved $5 \times 10^{6} \mathrm{HeLa}-\mathrm{CD} 4$ cells were pre-treated with CypA $(100 \mathrm{ng} / \mathrm{ml})$ or PMA (100 nM), and incubated 
at $37^{\circ} \mathrm{C}$ for various time periods. Cells were lysed in Laemmli sample buffer (BioRad, Hercules, CA), sonicated for 5-7 seconds to shear DNA and reduce sample viscosity, separated on 10-20\% SDS-PAGE and subjected to Western blotting analysis using anti-p44/p42 MAPK antibody and anti-phosphorylated-p44/p42 pMAPK antibody.

\section{Statistical analysis}

Data are presented as mean \pm S.E.M. Statistical analysis was performed using Student's t-test and $\mathrm{p}<0.05$ was considered significant.

\section{Results and discussion}

To manipulate CD147 expression levels on HeLa cells we used the RNAi knockdown approach. HeLa-CD4 (MAGI) cells were transfected with the pSuper-retro vector (OligoEngine, Seattle, WA) containing puromycin resistance gene and siRNA corresponding to the nucleotides 470-490 of CD147 mRNA. Puromycin resistant cells displayed lower concentrations of surface CD147 than parental cell line. The cells with the highest CD147 surface expression were removed using magnetic beads followed by flow cytometric cell sorting of remaining cells. Cell clones with decreased CD147 abundance were established from the remaining cell population. Cell clones with low and medium CD147 expression were selected for further analysis in comparison to original HeLa-CD4 cell clones transduced with an empty vector (high level CD147 expressors) (Figure 1A). Importantly, the level of expression changed after several passages of cells, making it necessary to verify the expression level before each experiment.

Using HeLa-CD4 cells transduced with an empty vector, we first performed a dose response analysis to identify the optimal concentration of CypA needed to induce chemotaxis. The concentration inducing maximal chemotactic response was $100 \mathrm{ng} / \mathrm{ml}$ and was used in all subsequent experiments. A good correlation between the level of CD147 expression and the chemotactic response of the cells to CypA was observed, with significant differences in chemotactic index between low, medium, and high expressing cells (Figure 1B). The observed chemotactic response was dependent on CD147 as it was completely blocked when anti-CD147 mAb was added to the cells in the upper chamber. Increased cell migration was not due to chemokinesis, as no migration was observed when CypA was added to both chambers of the Boyden chamber assembly. Importantly, the level of CD147 expression did not affect the ability of cells to migrate in response to SDF- $1 \alpha$ (Figure $1 C$ ), thus excluding the possibility that low CD147 adversely and non-specifically affected cell mobility. In fact, cells with low CD147 expression migrated to SDF-1 $\alpha$ better than high-CD147-expressing cells. This result may reflect some antagonism between these two receptors, suggested also by their opposing reaction to TCR activation in T lymphocytes [19]. These results are consistent with previously observed correlation between CD147 expression and CypA-induced chemotaxis of CD4+ T cells [6], however, in contrast to that previous study where activated and non-activated $\mathrm{T}$ cells were compared, siRNA treatment of the cells in this study is unlikely to affect expression of other than CD147 molecules, thus excluding the possibility that observed differences are due to altered expression of another signalling molecule involved in response to eCypA. Our data also indicate that a certain minimal level of CD147 expression is required for chemotactic response of cells to cyclophilin.

Cell chemotaxis is dependent on signalling events transduced by the chemotactic receptor in response to ligand binding. Interaction of cyclophilin with CD147 induces a number of signalling events, including $\mathrm{Ca}^{2+}$ mobilization and Erk 1/2 activation [3,9]. We analyzed CypA-induced Erk activation in HeLa cells expressing low and high levels of CD147. Consistent with results of the chemotaxis assay, cells with high level of CD147 expression exhibited Erk phosphorylation whereas Erk activation in cells with low level CD147 expression was minimal (Figure 2). Both high and low CD147 expressors equally responded to PMA activation, indicating that low response to eCypA was not due to an internal signalling defect. Despite the fact that the antibodies we used recognize both $\mathrm{p} 42$ and p44 forms of Erk, only one Erk form was detected in these experiments. This result may reflect the feature of the HeLa cell line where Erk 1 is expressed at a much lower level than Erk 2 [20]. This result establishes a correlation between the level of CD147 expression and Erk activation in response to eCypA stimulation.

Overall, results of this study provide evidence for the following two important features of the cellular responses to extracellular CypA. First, the response depends on the level of CD147 expression. Low CD147 expressing cells respond poorly to eCypA by chemotaxis or Erk activation. This dependence of response on CD147 abundance may be due to simple cumulative property of the signalling events, but may also reflect different modes of interaction between the ligand and receptor. For example, CD147, when present at low density on the cell surface, may not form homo-oligomers [21]. Although requirement of CD147 oligomerization for CypA-induced signalling has not been established, there is ample evidence for oligomerization of other chemotactic receptors [22-24]. Oligomerization of CD147 may be required to allow eCypA make contact with Proline 211 at the border of the transmembrane and ectodomain of CD147, which is the target of isomerase activity of CypA [25]. In any case, this result suggests that $\mathrm{CD} 147$ is the rate-limiting component of the multimeric receptor complex responding to eCypA. 

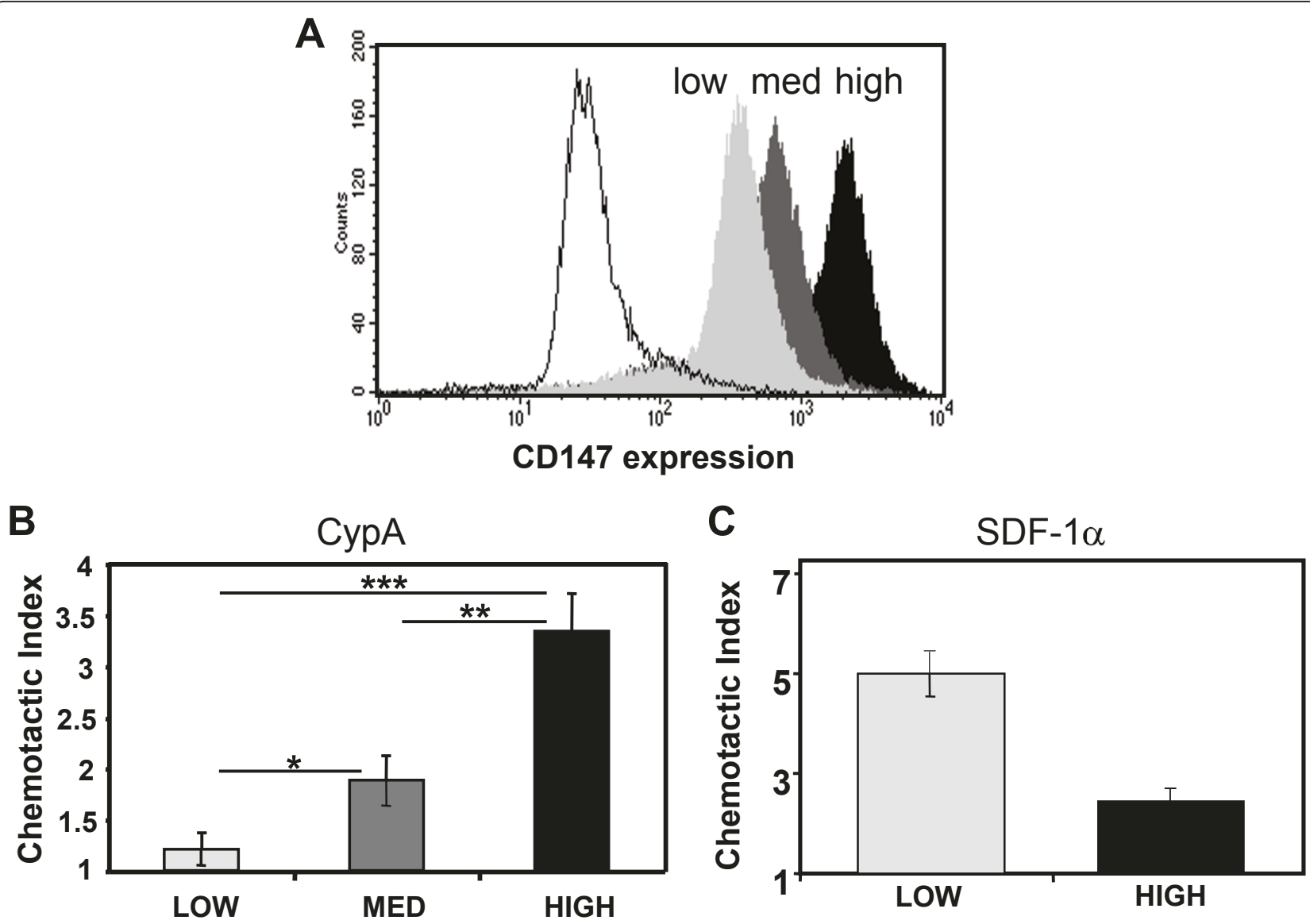

Figure 1 CD147 expression and chemotactic activity. HeLa-CD4 cells stably transfected with anti-CD147 siRNA-expressing vector or empty vector control were sorted according to the level of CD147 expression into low, medium and high (cells transfected with empty vector) expressing clones (A). Chemotactic activity of the clones in response to CypA (B) or SDF-1 $\alpha$ (C) was measured as described in Materials and Methods. Chemotactic activity was analyzed in triplicate and results are presented as mean \pm SEM, ${ }^{*} p<0.05 ;{ }^{* *} p<0.01$; ${ }^{* * *} p<0.001$. Results are shown for one representative experiment out of two performed.

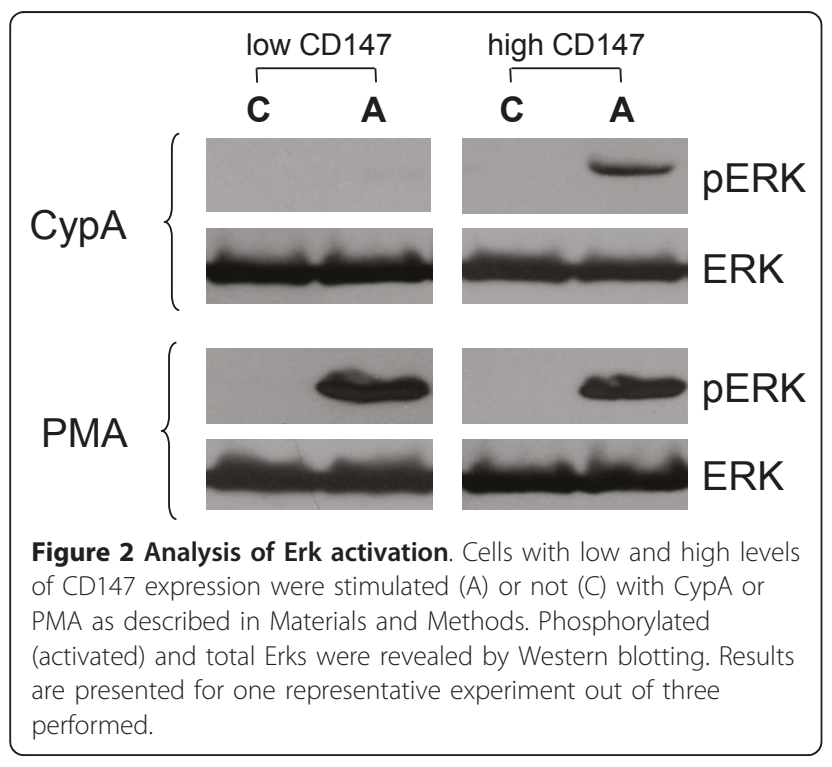

Another important conclusion from this study is that the chemotaxis-inducing activity of eCypA is not limited to leukocytes but likely extends to any cell type expressing sufficient levels of CD147. This feature would be especially relevant to cancer cells, which are known to express high CD147 levels (reviewed in [26]). CypAinduced chemotaxis may be an important contributor to metastatic activity of cancer cells. Correspondingly, targeting CD147 expression level may be a valid therapeutic approach aimed at reducing metastatic activity. One of the key regulators of the level of plasma membrane expression of CD147 is Cyp60 [27,28], a member of the cyclophilin family of proteins [29]. Expression of this protein in various cells has not been characterized, but may be an important factor determining cellular responses to eCyp and a potential target for therapeutic interventions. Another potential type of effector cells responsive to extracellular CypA are stem cells [15]. Additional studies are required to correlate the level of 
CD147 expression on different stem cells with their chemotactic activity or other responses.

\section{Conclusions}

Results of the current study provide the first demonstration of the chemotactic activity of HeLa cells to eCypA. They also establish a direct correlation between cellular chemotactic and signalling responses to extracellular cyclophilin A and the level of CD147 expression, suggesting that CD147 is the rate-limiting factor in the receptor complex responding to eCyp. These findings provide a strong rationale for investigating regulators of CD147 expression, which may be targeted to moderate eCypinduced cellular responses in inflammatory diseases and metastatic activity of cancer cells.

\section{Acknowledgements}

The authors acknowledge excellent technical assistance of Larisa Dubrovsky. Funded by R56 Al081152 and R01 Al067254 (SC and MB). The following reagent was obtained through the AIDS Research and Reference Reagent Program, Division of AIDS, NIAID, NIH: HeLa-CD4-LTR- $\beta$-gal from Dr. Michael Emerman.

\section{Author details}

${ }^{1}$ The George Washington University, Washington, DC 20037, USA. ${ }^{2} \mathrm{NHLBI}$, National Institutes of Health, Bethesda, MD 20892, USA. ${ }^{3} \mathrm{NCl}$, National Institutes of Health, Bethesda, MD 20892, USA.

\section{Authors' contributions}

All authors read and approved the final manuscript. AT carried out cell cloning; TP performed flow cytometry and Erk activation assays; SH participated in flow cytometry analysis of CD147 expression and performed chemotaxis assays; SC contributed to the overall design of the study and to manuscript preparation; BB designed the primers for siRNA expression and contributed to cell cloning; and MB designed the study and wrote the manuscript.

\section{Competing interests}

The authors declare that they have no competing interests.

Received: 16 August 2011 Accepted: 11 October 2011

Published: 11 October 2011

\section{References}

1. Fernandez EJ, Lolis E: Structure, function, and inhibition of chemokines. Annu Rev Pharmacol Toxicol 2002, 42:469-499.

2. Bukrinsky MI: Cyclophilins: unexpected messengers in intercellular communications. Trends Immunol 2002, 23:323-325.

3. Yurchenko V, Zybarth G, O'Connor M, Dai WW, Franchin G, Hao T, et al: Active site residues of cyclophilin $A$ are crucial for its signaling activity via CD147. J Biol Chem 2002, 277:22959-22965.

4. Arora K, Gwinn WM, Bower MA, Watson A, Okwumabua I, MacDonald HR, et al: Extracellular cyclophilins contribute to the regulation of inflammatory responses. J Immunol 2005, 175:517-522.

5. Gwinn WM, Damsker J, Falahati R, Okwumabua I, Kelly-Welsh A, Keegan A, et al: Novel approach to inhibit asthma-mediated lung inflammation using anti-CD147 intervention. J Immunol 2006, 177:4870-4879.

6. Damsker JM, Bukrinsky Ml, Constant SL: Preferential chemotaxis of activated human CD4+ T cells by extracellular cyclophilin A. J Leukoc Biol 2007, 82:613-618.

7. Sherry B, Yarlett N, Strupp A, Cerami A: Identification of cyclophilin as a proinflammatory secretory product of lipopolysaccharide-activated macrophages. Proc Natl Acad Sci USA 1992, 89:3511-3515.

8. Xu Q, Leiva MC, Fischkoff SA, Handschumacher RE, Lyttle CR: Leukocyte chemotactic activity of cyclophilin. J Biol Chem 1992, 267:11968-11971.
9. Yurchenko V, O'Connor M, Dai WW, Guo H, Toole B, Sherry B, et al: CD147 is a signaling receptor for cyclophilin B. Biochem Biophys Res Commun 2001, 288:786-788.

10. Pushkarsky T, Yurchenko V, Laborico A, Bukrinsky M: CD147 stimulates HIV1 infection in a signal-independent fashion. Biochem Biophys Res Commun 2007, 363:495-499

11. Berditchevski F, Chang S, Bodorova J, Hemler ME: Generation of monoclonal antibodies to integrin-associated proteins. Evidence that alpha3beta1 complexes with EMMPRIN/basigin/OX47/M6. J Biol Chem 1997, 272:29174-29180

12. Cho JY, Fox DA, Horejsi V, Sagawa K, Skubitz KM, Katz DR, et al: The functional interactions between CD98, \{beta\}1-integrins, and CD147 in the induction of U937 homotypic aggregation. Blood 2001, 98:374-382.

13. Melchior A, Denys A, Deligny A, Mazurier J, Allain F: Cyclophilin B induces integrin-mediated cell adhesion by a mechanism involving CD98dependent activation of protein kinase C-delta and p44/42 mitogenactivated protein kinases. Exp Cell Res 2008, 314:616-628.

14. Pakula R, Melchior A, Denys A, Vanpouille C, Mazurier J, Allain F: Syndecan$1 / C D 147$ association is essential for cyclophilin B-induced activation of p44/42 mitogen-activated protein kinases and promotion of cell adhesion and chemotaxis. Glycobiology 2007, 17:492-503.

15. Attia M, Huet E, Delbe J, Ledoux D, Menashi S, Martelly I: Extracellular matrix metalloproteinase inducer (EMMPRIN/CD147) as a novel regulator of myogenic cell differentiation. J Cell Physiol 2011, 226:141-149.

16. Hao JL, Cozzi PJ, Khatri A, Power CA, Li Y: CD147/EMMPRIN and CD44 are potential therapeutic targets for metastatic prostate cancer. Curr Cancer Drug Targets 2010, 10:287-306.

17. Biswas C, Zhang Y, DeCastro R, Guo H, Nakamura T, Kataoka H, et al: The human tumor cell-derived collagenase stimulatory factor (renamed EMMPRIN) is a member of the immunoglobulin superfamily. Cancer Res 1995, 55:434-439.

18. Kimpton J, Emerman M: Detection of replication-competent and pseudotyped human immunodeficiency virus with a sensitive cell line on the basis of activation of an integrated beta-galactosidase gene. $J$ Virol 1992, 66:2232-2239.

19. Ganor $Y$, Teichberg VI, Levite M: TCR activation eliminates glutamate receptor GluR3 from the cell surface of normal human T cells, via an autocrine/paracrine granzyme B-mediated proteolytic cleavage. $J$ Immunol 2007, 178:683-692.

20. Heider H, Hug C, Lucocq JM: A 40-kDa myelin basic protein kinase, distinct from erk1 and erk2, is activated in mitotic HeLa cells. Eur $\rfloor$ Biochem 1994, 219:513-520.

21. Yoshida S, Shibata M, Yamamoto S, Hagihara M, Asai N, Takahashi M, et al: Homo-oligomer formation by basigin, an immunoglobulin superfamily member, via its N-terminal immunoglobulin domain. Eur J Biochem 2000, 267:4372-4380

22. Hamatake M, Aoki T, Futahashi $\mathrm{Y}$, Urano $\mathrm{E}$, Yamamoto $\mathrm{N}$, Komano J: Ligand-independent higher-order multimerization of CXCR4, a Gprotein-coupled chemokine receptor involved in targeted metastasis. Cancer Sci 2009, 100:95-102.

23. Handel TM, Johnson Z, Rodrigues DH, Dos Santos AC, Cirillo R, Muzio V, et al: An engineered monomer of CCL2 has anti-inflammatory properties emphasizing the importance of oligomerization for chemokine activity in vivo. J Leukoc Biol 2008, 84:1101-1108.

24. Wang J, He L, Combs CA, Roderiquez G, Norcross MA: Dimerization of CXCR4 in living malignant cells: control of cell migration by a synthetic peptide that reduces homologous CXCR4 interactions. Mol Cancer Ther 2006, 5:2474-2483.

25. Schlegel J, Redzic JS, Porter CC, Yurchenko V, Bukrinsky M, Labeikovsky W, et al: Solution characterization of the extracellular region of CD147 and its interaction with its enzyme ligand cyclophilin A.J Mol Biol 2009, 391:518-535.

26. Weidle UH, Scheuer W, Eggle D, Klostermann S, Stockinger H: Cancerrelated issues of CD147. Cancer Genomics Proteomics 2010, 7:157-169.

27. Yurchenko V, Pushkarsky T, Li JH, Dai WW, Sherry B, Bukrinsky M: Regulation of CD147 cell surface expression: involvement of the proline residue in the CD147 transmembrane domain. J Biol Chem 2005, 280:17013-17019.

28. Pushkarsky T, Yurchenko V, Vanpouille C, Brichacek B, Vaisman I, Hatakeyama S, et al: Cell surface expression of CD147/EMMPRIN is regulated by cyclophilin 60. J Biol Chem 2005, 280:27866-27871. 
29. Wang BB, Hayenga KJ, Payan DG, Fisher JM: Identification of a nuclearspecific cyclophilin which interacts with the proteinase inhibitor eglin $\mathrm{c}$. Biochem J 1996, 314(Pt 1):313-319.

doi:10.1186/1756-0500-4-396

Cite this article as: Trachtenberg et al: The level of CD147 expression correlates with cyclophilin-induced signalling and chemotaxis. BMC Research Notes 2011 4:396.

Submit your next manuscript to BioMed Central and take full advantage of:

- Convenient online submission

- Thorough peer review

- No space constraints or color figure charges

- Immediate publication on acceptance

- Inclusion in PubMed, CAS, Scopus and Google Scholar

- Research which is freely available for redistribution 